\title{
\begin{tabular}{l|l} 
Mitraries & DSpace@MIT
\end{tabular}
}

\author{
MIT Open Access Articles
}

\section{Reservoir characterization in an underground gas storage field using joint inversion of flow and geodetic data}

The MIT Faculty has made this article openly available. Please share how this access benefits you. Your story matters.

Citation: Jha, B., F. Bottazzi, R. Wojcik, M. Coccia, N. Bechor, D. McLaughlin, T. Herring, B. H. Hager, S. Mantica, and R. Juanes. "Reservoir Characterization in an Underground Gas Storage Field Using Joint Inversion of Flow and Geodetic Data." International Journal for Numerical and Analytical Methods in Geomechanics 39, no. 14 (August 26, 2015): 1619-1638.

As Published: http://dx.doi.org/10.1002/nag.2427

Publisher: Wiley Blackwell

Persistent URL: http://hdl.handle.net/1721.1/98903

Version: Author's final manuscript: final author's manuscript post peer review, without publisher's formatting or copy editing

Terms of use: Creative Commons Attribution-Noncommercial-Share Alike 


\title{
Reservoir characterization in an underground gas storage field using joint inversion of flow and geodetic data
}

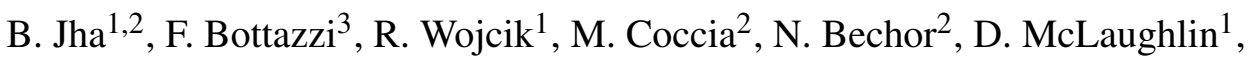 \\ T. Herring ${ }^{2}$, B. H. Hager ${ }^{2}$, S. Mantica ${ }^{3}$ and R. Juanes ${ }^{1,2, *, \dagger}$ \\ ${ }^{1}$ Civil and Environmental Engineering, Massachusetts Institute of Technology, Cambridge, MA, U.S.A \\ ${ }^{2}$ Earth, Atmospheric and Planetary Sciences, Massachusetts Institute of Technology, Cambridge, MA, U.S.A. \\ ${ }^{3}$ Divisione E\&P, Eni S.p.A., San Donato Milanese, Italy
}

\begin{abstract}
SUMMARY
Characterization of reservoir properties like porosity and permeability in reservoir models typically relies on history matching of production data, well pressure data, and possibly other fluid-dynamical data. Calibrated (history-matched) reservoir models are then used for forecasting production and designing effective strategies for improved oil and gas recovery. Here, we perform assimilation of both flow and deformation data for joint inversion of reservoir properties. Given the coupled nature of subsurface flow and deformation processes, joint inversion requires efficient simulation tools of coupled reservoir flow and mechanical deformation. We apply our coupled simulation tool to a real underground gas storage field in Italy. We simulate the initial gas production period and several decades of seasonal natural gas storage and production. We perform a probabilistic estimation of rock properties by joint inversion of ground deformation data from geodetic measurements and fluid flow data from wells. Using an efficient implementation of the ensemble smoother as the estimator and our coupled multiphase flow and geomechanics simulator as the forward model, we show that incorporating deformation data leads to a significant reduction of uncertainty in the prior distributions of rock properties such as porosity, permeability, and pore compressibility. Copyright $\odot$ 2015 John Wiley \& Sons, Ltd.
\end{abstract}

Received 2 February 2015; Revised 13 July 2015; Accepted 3 August 2015

KEY WORDS: inversion; ensemble Kalman; geomechanics; InSAR; subsidence

\section{INTRODUCTION}

Reservoir characterization refers to the estimation of spatial distributions of rock and fluid properties in the reservoir, such as porosity, permeability, and compressibility. These properties control the changes in the state of the reservoir-fluid saturations, pore pressures, displacements, and stressdue to production and injection through wells. Dynamic reservoir simulation models are utilized to evaluate such changes in the reservoir. Reservoir characterization is an important step during the construction of a dynamic reservoir model and its subsequent refinement by integration of data recorded during the development of the field. While the initial characterization of reservoir properties like porosity and permeability is based on well log data and assumed inter-well statistics of these properties, history matching with well rate and pressure data during reservoir simulation further constrains the porosity and permeability distributions in the reservoir [1]. History-matched reservoir flow models, which are traditionally uncoupled from mechanical deformation of the reservoir, are then used for forecasting production and designing effective strategies for improved oil and gas recovery. However, history matching is often non-unique [1-3]. Other than nonlinearity of the

*Correspondence to: R. Juanes, Civil and Environmental Engineering, Massachusetts Institute of Technology, Cambridge, MA, U.S.A.

${ }^{\dagger}$ E-mail: juanes@mit.edu 
dynamic model, one of the reasons for this non-uniqueness is that the number of unknown reservoir parameters in the model is typically much greater than the number of informative measurements at the wells. In addition, the flow response of a reservoir depends on both fluid flow and mechanical deformation, which are coupled processes in the subsurface. This suggests that by acquiring and assimilating geodetic data, in addition to flow data at the wells, it might be possible to reduce the uncertainty in reservoir parameters.

As the amount and type of data available during the field development increase, there is a need for novel techniques that can incorporate the newly available data in the characterization process. For example, with the advent of time-lapse seismic monitoring [4], joint inversion of flow and seismic data has emerged as a viable reservoir characterization technique aimed at reducing uncertainty in rock physics models [5-8]. Recently, it has been shown that ground deformation data available from geodetic measurements, such as Global Positioning System (GPS) and interferometric synthetic aperture radar (InSAR), can be used as a valuable diagnostic to study reservoir compaction and subsidence due to groundwater withdrawal [9,10], geologic storage of carbon dioxide [11], and seasonal ground motion due to storage and withdrawal of gas from depleted gas reservoirs [12]. In the study of natural and induced earthquakes, ground deformation data are also being used to estimate the location and sense of slip on earthquake-inducing faults [13]. Using a joint inversion analysis, surface deformation data have been used in conjunction with well pressure and/or flow rate data to estimate subsurface properties such as permeability fields [14-17], bulk compressibility [18], and fault transmissibilities [19].

In this paper, we perform joint inversion of flow and surface deformation data for statistical estimation of rock properties in a real underground gas storage (UGS) field with complex geology and production history, pseudo-named as the beta reservoir. We show that it is possible to reduce uncertainty in estimates of these properties by assimilating both sources of data in a one-way coupled flow and geomechanics formulation. The coupled formulation, which is described elsewhere [20], provides the forward model for the inversion.

\section{THE BETA RESERVOIR DATASET}

The analysis described in the present paper has been conducted on a real gas storage field, which will be referred to as the beta field. The beta field is composed of three units, named beta-1, beta-2, and beta-3, located at three different depths. In the present work, only the main reservoir, Beta3 , has been considered for the analysis, because the three units are hydraulically separated, and it has been verified that the contributions of beta- 1 and beta- 2 are minimal compared with those of beta-3 in terms of induced displacement at the ground surface level. Beta-3 is located at around $1300-\mathrm{m}$ depth and is composed of sandy layers. It is connected with a large and strong aquifer in the southwest direction. The field produced for a few years before the UGS project started, in which methane is injected into the reservoir from April to October and produced from November to March. Ground surface motion above the reservoir follows this seasonal cycle of storage and withdrawal. During the injection months, the surface is uplifted vertically with expansion in the eastwest and north-south directions away from the points of injection. During the production months, the ground surface subsides vertically with contraction in the horizontal directions. The datasets for the beta field inversion are the well bottomhole pressure (WBHP) for all producing/injecting wells and the line-of-sight (LOS) surface displacements over the field as measured by SAR sensors on the Envisat satellite. The spatial coverage and temporal coverage of the two types of data are different. Moreover, the pressure dataset covers two types of flow regimes: a depletion regime during pre-UGS period when the pressure declines monotonically and the subsequent pseudo steady-state regime during the UGS period when the pressure fluctuates in response to injection and production. The InSAR data contain phase changes associated with ground deformation in the LOS direction of the radar beam (Figure 1). To translate to displacements, we multiply the phase changes by $\lambda / 4 \pi$, where $\lambda$ is the wavelength of the radar beam [21]. We process the raw InSAR images using the persistent scatterer (PS) approach [22]. Next, we remove small-scale random noise and large-scale signals that are not due to UGS-induced reservoir deformation, such as signals due to atmospheric 


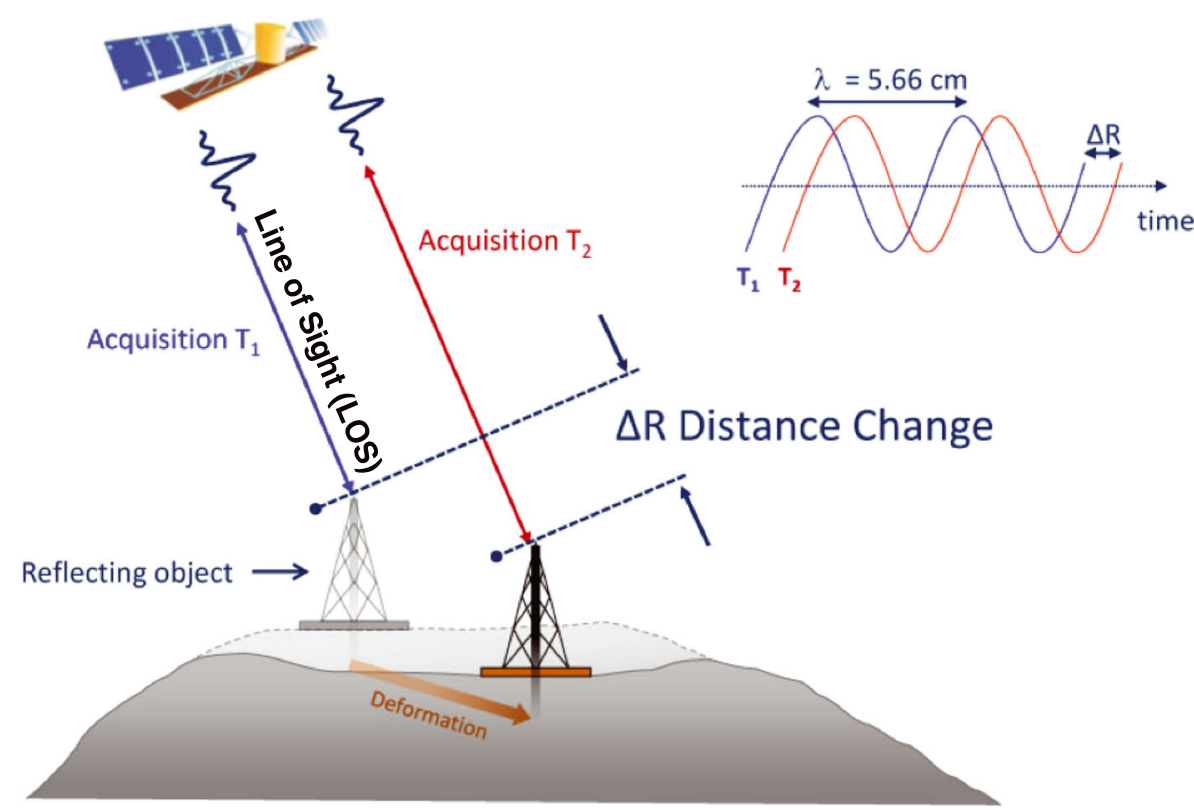

Figure 1. Schematic of interferometric synthetic aperture radar data acquisition using an earth-orbiting satellite equipped with synthetic aperture radar sensors. The angles between the satellite's line of sight and the 3D co-ordinate directions-east, north, and upward-are used to convert displacement vectors from a 3D geomechanical simulation into line-of-sight displacement values. Image courtesy of Tele-Rilevamento Europe [23].

features, orbital errors, regional geological events, and regional subsidence. Removal of small-scale noise reduces the computational cost of inversion by reducing the number of assimilation points.

\subsection{Interferometric synthetic aperture radar data processing}

Satellite-based radar interferograms contain spatially correlated signals, mostly related to atmospheric and ionospheric structures that are commonly coherent over length scales of tens to hundreds of kilometers [24-26]. Correlation between range change and topography results from the variation of the refractivity of the atmosphere along the vertical due to changes in pressure, temperature, humidity, and water vapor content in the lowermost atmosphere between two SAR acquisitions [26]. For a vertically stratified troposphere model, if elevation changes across the scene, propagation delays vary at different elevations with a rate increasing with water vapor content and the pressuretemperature ratio [27]. We characterize these large-scale signals in our InSAR images with a covariance analysis.

Assuming that the noise is spatially stationary and isotropic, the covariance between any two points $\boldsymbol{x}=(x, y)$ and $\boldsymbol{x}+\boldsymbol{r}$ depends only on the distance $r=|\boldsymbol{r}|$ between them, that is, $\operatorname{Cov}(\boldsymbol{x}, \boldsymbol{x}+$ $\boldsymbol{r})=\operatorname{Cov}(r)$. We calculate experimental covariance of the PS, using all the data that do not contain deformation, that is, outside the gas reservoir area. We subtract the best-fitting phase plane to remove a potential error ramp caused by inaccurate orbital information. We calculate a sample covariance function for the interferogram images as follows. For each distance $r$ in an image at timestep $t$, we select many random locations $\left(x_{i}, y_{i}\right)$ and directions $\theta_{i}$ within the image and compare the value at $\left(x_{i}, y_{i}\right)$ and $\left(x_{i}+r \cos \theta_{i}, y_{i}+r \sin \theta_{i}\right)$ to calculate the sample covariance curve of the image. We fit a normalized Gaussian covariance function, $\frac{1}{L_{a, t} \sqrt{2 \pi}} \exp \left(-r^{2} / 2 L_{a, t}^{2}\right)$, to the sample covariance curve, where $L_{a, t}$ is the correlation length in the large-scale signal at timestep $t$. The value of $L_{a, t}$ is approximately 10 to $50 \mathrm{~km}$. We calculate average displacements on a $2 \mathrm{D}$ grid with a grid cell size based on $L_{a, t}$, and we subtract the average from the image to remove the large-scale signal. Next, we apply a 2D Gaussian smoothing filter in each grid cell based on the average and standard deviation of displacements within the cell. The filter removes the high-frequency noise by setting each point's displacement to a weighted average of displacements in that point's neighborhood. This reduces the 
number of assimilation points in the inversion. Finally, we calculate the covariance of the filtered images and estimate new correlation lengths, $L_{c, t}$, which are representative of the UGS-induced deformation. In our dataset, $L_{c, t}$ varies from 2 to $10 \mathrm{~km}$.

\section{THE JOINT INVERSION MODEL}

In this study, inversion is the process of estimating unknown subsurface parameters such as porosity, permeability, and rock compressibility by assimilating measurements of ground displacements, well rates, and pressures with predictions from a forward model. The forward model in our case is a one-way coupled flow and geomechanics model, which we simulate using our coupled flow and geomechanics simulator, PYLITH-GPRS [20].

We use an ensemble-based data assimilation method for statistical estimation. Data assimilation methods offer the following advantages over optimization-based approaches [28, 29]: (i) they are based on a Bayesian framework to capture uncertainty propagation and, hence, can generate many history-matched models simultaneously; (ii) they do not require derivation of gradient operators or adjoint equations with backward-in-time integration and, hence, are independent of the forward model simulator; and (iii) they allow sequential and forward-in-time update of history-matched models with sequentially available measurements and, hence, avoid calibration to the entire history every time a new measurement becomes available.

Usually, the measurements are available at different points in space and time, whereas the rock parameters are assumed to vary only in space. Assimilating measurements from multiple points in space and time provides robustness to the inversion process and increases confidence in the estimates [14]. Uncertainty in physically related parameters is often modeled as a multi-Gaussian random process defined with the parameter mean and covariance, although non-Gaussian contributions arising from nonlinear dynamics have also been considered [30, 31]. The Gaussian assumption provides analytical tractability and uniqueness to the solution of the inversion problem, albeit at the cost of accuracy in the representation of non-Gaussian variations in the parameters.

The Kalman filter is a linear unbiased estimator to update prior estimates of the model parameters of a dynamical model by assimilating predictions and measurements of the model state variables sequentially at different timesteps [32-34]. It is the optimal least square estimator under the assumptions of linear dynamics, unbiased and Gaussian prior statistics, Gaussian measurement error, and no correlation between the error in the prior ensemble and the measurement error. The ensemble Kalman filter (EnKF) is a Monte Carlo approximation of the Kalman filter suitable for large-scale, possibly nonlinear, problems with many unknowns such as the ones encountered in geophysical applications [28, 29, 35-38] (see Figure 2 for a general idea). Similar to many ensemble methods, it only uses the first two statistical moments, mean and covariance, of the prior distributions of the model parameters and errors to update the prior ensemble. It requires the ensemble

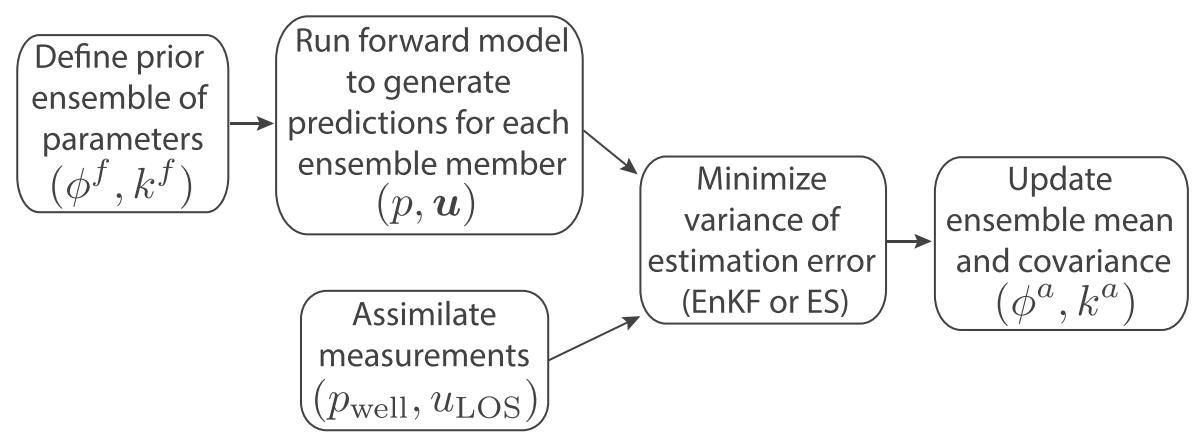

Figure 2. A generic flow chart for joint inversion using ensemble Kalman methods. $\phi^{f}$ and $k^{f}$ are the first-guess or prior values of porosity and permeability, respectively. $\phi^{a}$ and $k^{a}$ are the analyzed or posterior values of porosity and permeability, respectively. $p$ and $\boldsymbol{u}$ are the predicted pore pressure and displacement vector, respectively. $p_{\text {well }}$ and $u_{\text {LOS }}$ are the measured well pressure and line-of-sight displacement, respectively. 
covariance matrix of the prior distributions, measurements of the state variables and their error covariance matrix, a forward model to generate predictions of the measurements, and a mapping of the predictions to measurement locations. Simplification in the EnKF comes from the assumption that the true covariance matrix of the model parameters can be substituted with the ensemble covariance matrix when the ensemble is a large enough sample drawn from the prior distributions of the parameters.

\subsection{Ensemble smoother}

In a sequential data assimilation method such as EnKF, updating of the model parameters due to assimilation at a timestep requires stopping and restarting of the forward model time integration at each assimilation step for each realization. This is either carried out manually or requires coding of the data assimilation routine inside the forward model simulator. To avoid multiple restarts of the forward model every time measurements are available, an alternative method, the ensemble smoother (ES), is utilized [39, 40]. The ES method approximates the EnKF by assimilating all the past measurements in one update step. Because all timesteps are considered at once, the computational cost of the ES update is larger than that of a single update in EnKF. However, ES offers an advantage in the implementation cost of the assimilation process because it can be performed outside the forward model simulator, as a post-processing step. The disadvantage is that integration of a nonlinear model over a long time window, unconstrained by measurements, introduces non-Gaussian contributions in the statistics [28]. These contributions are not captured in the Kalman methods and, therefore, can lead to error in the estimates. ES is a useful method to estimate the state parameters when these parameters do not change with time during the assimilation period. Recently, ES has been proposed as an efficient history-matching method for oil and gas reservoirs because of the diffusive nature of the solution [40].

We use the ES $[40,41]$ for estimation and uncertainty reduction of model parameters in the beta field. We choose the model parameters in our study based on the following criteria: importance of a parameter in determining the surface displacement due to UGS operations, effect of assimilating two types of data (well pressure and InSAR displacements) in reducing uncertainty in a parameter, and prior uncertainty in a parameter in the beta field. Here, we provide a brief introduction to the ES method. Let $n$ be the number of model parameters, $q$ be the number of state variables or model output variables, $m$ be the number of measurements, and $N$ be the number of members in the ensemble. Neglecting the uncertainty in the forward model, which is assumed to be deterministic, we have updated the ensemble of the model parameters as follows [41, 42]:

$$
\boldsymbol{\alpha}^{a}=\boldsymbol{\alpha}^{f}+\boldsymbol{C}_{\alpha d}\left(\boldsymbol{C}_{d d}+\boldsymbol{C}_{\epsilon \epsilon}\right)^{-1}\left(\boldsymbol{d}-\boldsymbol{d}^{f}\right),
$$

where $\boldsymbol{\alpha}^{f} \in \mathcal{R}^{n \times N}$ is the first-guess or prior ensemble, $\boldsymbol{\alpha}^{a} \in \mathcal{R}^{n \times N}$ is the updated or posterior ensemble of the model parameters, $\boldsymbol{d} \in \mathcal{R}^{m \times N}$ is the measurement matrix containing perturbed measurements of the state variables, $\boldsymbol{d}^{f} \in \mathcal{R}^{m \times N}$ is the predicted measurements, $\boldsymbol{C}_{\epsilon \epsilon} \in \mathcal{R}^{m \times m}$ is the ensemble covariance matrix of measurement errors, $\boldsymbol{C}_{d d} \in \mathcal{R}^{m \times m}$ is the ensemble covariance matrix of the model predicted measurements, and $\boldsymbol{C}_{\alpha d} \in \mathcal{R}^{n \times m}$ is the cross-covariance matrix between the prior model parameters and the predicted measurements. The perturbed measurement matrix $\boldsymbol{d}$ is assumed to be Gaussian and created by applying the standard Gaussian perturbation to the measurement vector $\boldsymbol{d}_{m} \in \mathcal{R}^{m}$. We estimate the prediction covariance matrix as $C_{d d}=\left(\boldsymbol{d}^{f}-\overline{\boldsymbol{d}^{f}}\right)\left(\boldsymbol{d}^{f}-\overline{\boldsymbol{d}^{f}}\right)^{T} /(N-1)$ and the cross-covariance matrix as $\boldsymbol{C}_{\alpha d}=\left(\boldsymbol{\alpha}^{f}-\overline{\boldsymbol{\alpha}^{f}}\right)\left(\boldsymbol{d}^{f}-\overline{\boldsymbol{d}^{f}}\right)^{T} /(N-1)$. Measurements are predicted using the forward model, $\boldsymbol{d}^{f}=\mathcal{M}\left[\boldsymbol{G}\left(\boldsymbol{\alpha}^{f}\right)\right]$, where $\boldsymbol{G}$ is the nonlinear forward model operator, $\boldsymbol{G}\left(\boldsymbol{\alpha}^{f}\right) \in \mathcal{R}^{q \times N}$ is the model prediction of the state variables, and $\mathcal{M}$ is the measurement functional that projects model prediction onto measurement locations where $\boldsymbol{d}_{m}$ is sampled, such that $\mathcal{M}\left[\boldsymbol{G}\left(\boldsymbol{\alpha}^{f}\right)\right] \in \mathcal{R}^{m \times N}$ is the projected prediction.

It follows from Equation (1) that the model parameters are updated based on the weighted mismatch between measurements and predictions of state variables at prescribed locations. The weights, which are derived from the inverse of the error covariances or confidence in the measurements, are 
contained in the prefactor $\boldsymbol{C}_{\alpha d}\left(\boldsymbol{C}_{d d}+\boldsymbol{C}_{\epsilon \epsilon}\right)^{-1}$, also known as the Kalman gain matrix. Reduction in uncertainty of a parameter depends on the sensitivity of the state variables, such as pressure and displacement, to that parameter. Different parameters may experience different degrees of uncertainty reduction. These sensitivities are included in the forward model through the governing equations of the physical system. In the ES framework, uncertainty reduction due to ensemble update in Equation (1) can be expressed through the covariance update equation [28],

$$
C_{\alpha \alpha}^{a}=C_{\alpha \alpha}^{f}-C_{\alpha d}\left(C_{d d}+C_{\epsilon \epsilon}\right)^{-1} C_{\alpha d}^{T},
$$

where the Kalman gain captures the sensitivity inherent in the forward model. One of the advantages of using ensemble methods such as ES is to replace the costly calculation of the sensitivity of state variables, which are needed in gradient-based parameter estimation methods, with a simpler algorithm to calculate the Kalman gain.

The standard ensemble Kalman analysis scheme requires random perturbations of measurements $\boldsymbol{d}_{m}$ to generate the ensemble measurement error covariance matrix $\boldsymbol{C}_{\epsilon \epsilon}$. It has been shown that this step can lead to sampling errors in the ensemble variance update equation, Equation (2), because of a finite ensemble size, and thus should be avoided in favor of other techniques such as the square root analysis scheme $[42,43]$. Here, we use the square root analysis scheme to avoid random perturbations to the measurement and invert $\boldsymbol{C}_{d d}+\boldsymbol{C}_{\epsilon \epsilon}$ using a fast reduced-rank approximation to singular value decomposition based on random matrix theory [44].

\section{THE FORWARD MODEL}

A forward model generates a prediction of quantities of interest for a given realization of the model parameters. In this study, the forward model is a one-way coupled flow and geomechanics model of the beta field. The model construction is divided into three stages: construction of the geological model, construction of the flow model, and construction of the geomechanical model. A geological model provides the basic architecture of the study area in terms of its structural (faults, folds, pinch-outs, etc.) and stratigraphic (sand-shale layering, lithology, etc.) features. Some of these features may act as barriers/conduits to flow and may have load-bearing capacity or deformation mode (such as elastic versus plastic) significantly different from the surrounding medium. In this way, a geological model provides an essential input during the construction of flow and geomechanical models. A geological model of the beta field is built from stratigraphic maps prepared from a 3D seismic survey and borehole logs available in the field. Later, we describe the flow and geomechanical models.

\subsection{Flow model}

The flow model has three main components: the gas reservoir, the aquifer, and the wells. The flow model is extended horizontally more than $20 \mathrm{~km}$ in the southwest direction to include the aquifer connected to the gas reservoir (Figure 3). This ensures that propagation of well-induced pressure

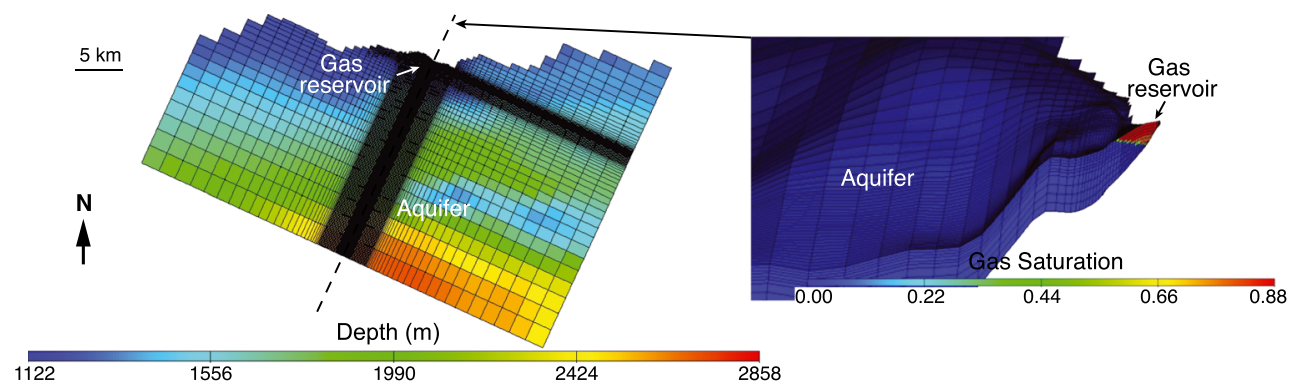

Figure 3. Top view (left) and a cross-section view along the dotted line (right) of the flow model grid. Left: the size of grid cells increases continuously from a small value in the gas reservoir to a large value in the aquifer. Right: initial gas saturation is plotted with red color indicating the maximum gas saturation. Gas accumulates near the top of the reservoir because of buoyancy. Wells (not shown in the image) are drilled into the gas reservoir. 
Table I. Model parameters used in the beta field inversion study.

\begin{tabular}{lcrrrr}
\hline Parameter & Distribution & Mean & Std. Dev. & Min & Max \\
\hline Reservoir porosity, $\phi$ & Normal & 0.292 & 0.011 & 0.264 & 0.327 \\
Poisson's ratio, $v$ & Normal & 0.300 & 0.006 & 0.286 & 0.316 \\
Compressibility intercept $a$ in Equation $(3)$ & Normal & -1.863 & 0.230 & -2.434 & -1.331 \\
Reservoir horizontal permeability, $\log k h_{r}$ & Normal & 2.596 & 0.029 & 2.534 & 2.676 \\
Vertical-to-horizontal permeability ratio, $k v_{r} / k h_{r}$ & Uniform & 0.902 & 0.055 & 0.804 & 1.000 \\
Aquifer-to-reservoir permeability ratio, $k_{a} / k_{r}$ & Uniform & 0.543 & 0.255 & 0.114 & 1.000 \\
\hline
\end{tabular}

All parameters are dimensionless except the log of horizontal permeability, which has the unit of $\log$ (millidarcy). Std. Dev., standard deviation.

perturbations through the reservoir and the surrounding region is correctly captured during the flow simulation. The areal extension of the model, covering both the gas-bearing region and the aquifer region, is $56 \times 54 \mathrm{~km}^{2}$. We discretize the flow domain with 10 layers in the vertical direction such that the average element thickness varies from 8 to $5 \mathrm{~m}$ in the gas-bearing region up to $25 \mathrm{~m}$ in the far aquifer region. The average dimension of elements in the lateral direction varies from $50 \mathrm{~m}$ in the gas-bearing region to $2 \mathrm{~km}$ in the aquifer region far from the wells, where pressure changes are expected to be small. The finite volume flow grid is composed of $139 \times 82 \times 10$ elements in a logically Cartesian grid, with directions roughly corresponding to east-west, north-south, and vertical, respectively.

The flow model is initialized with the following petrophysical and fluid properties: fluid properties (density, viscosity, and compressibility as functions of pressure), porosity, horizontal and vertical reservoir permeability, horizontal and vertical aquifer permeability, net-to-gross ratio (hydrocarbon-bearing fraction of the total vertical thickness), pore compressibility, relative permeability curves, gas-water contact depth, initial equilibrium pressure, and irreducible water saturations. The total compressibility that determines the transient response of the flow model combines both the pore compressibility and the fluid compressibility. Homogeneity is assumed for porosity, horizontal and vertical reservoir permeability, and horizontal and vertical aquifer permeability. Pore compressibility is assumed to be depth dependent. We keep these properties fixed during the simulation.

There is a relatively large uncertainty in the pressure support received from the aquifer during pressure fluctuations within the gas reservoir. This uncertainty stems from lack of fluid-dynamical and petrophysical data in the aquifer region, which has no wells. Aquifer pore volume and aquifer permeability are the two main parameters that determine the strength of the aquifer. In this study, we consider porosity, horizontal reservoir permeability, vertical-to-horizontal reservoir permeability ratio, and aquifer-to-reservoir permeability ratio as uncertain parameters in the flow model (Table I).

All the boundaries of the reservoir are designated as no-flow boundaries. The flow simulation is driven with gas production and injection rates available as measurements at wellheads (Figure 4). The flow simulation is conducted using the software general purpose research simulator (GPRS), which is a finite volume, multiphase, multicomponent petroleum reservoir simulation tool [20, 45]. The two key output quantities obtained from the flow simulation are the pressure field over the entire reservoir and the bottomhole pressure in each well at each timestep.

\subsection{Geomechanical model}

Starting from the fluid-dynamical model described earlier, a geomechanical model is built to simulate the surface displacement induced by reservoir exploitation and the UGS program. The flow model is extended both vertically and horizontally to account for overburden, underburden, and sideburden during reservoir deformation. The model is extended upwards to the ground surface, downwards to a depth of $5000 \mathrm{~m}$, and laterally for $20 \mathrm{~km}$ in both east-west and north-south directions. A geomechanical model larger than the flow model also allows us to correctly apply the boundary conditions of zero horizontal displacement on the four lateral boundaries, zero displacement on the bottom boundary, and a traction-free condition on the top boundary. The dimension of the geomechanical model is $90 \times 90 \times 5 \mathrm{~km}^{3}$. The overburden and the underburden are discretized 

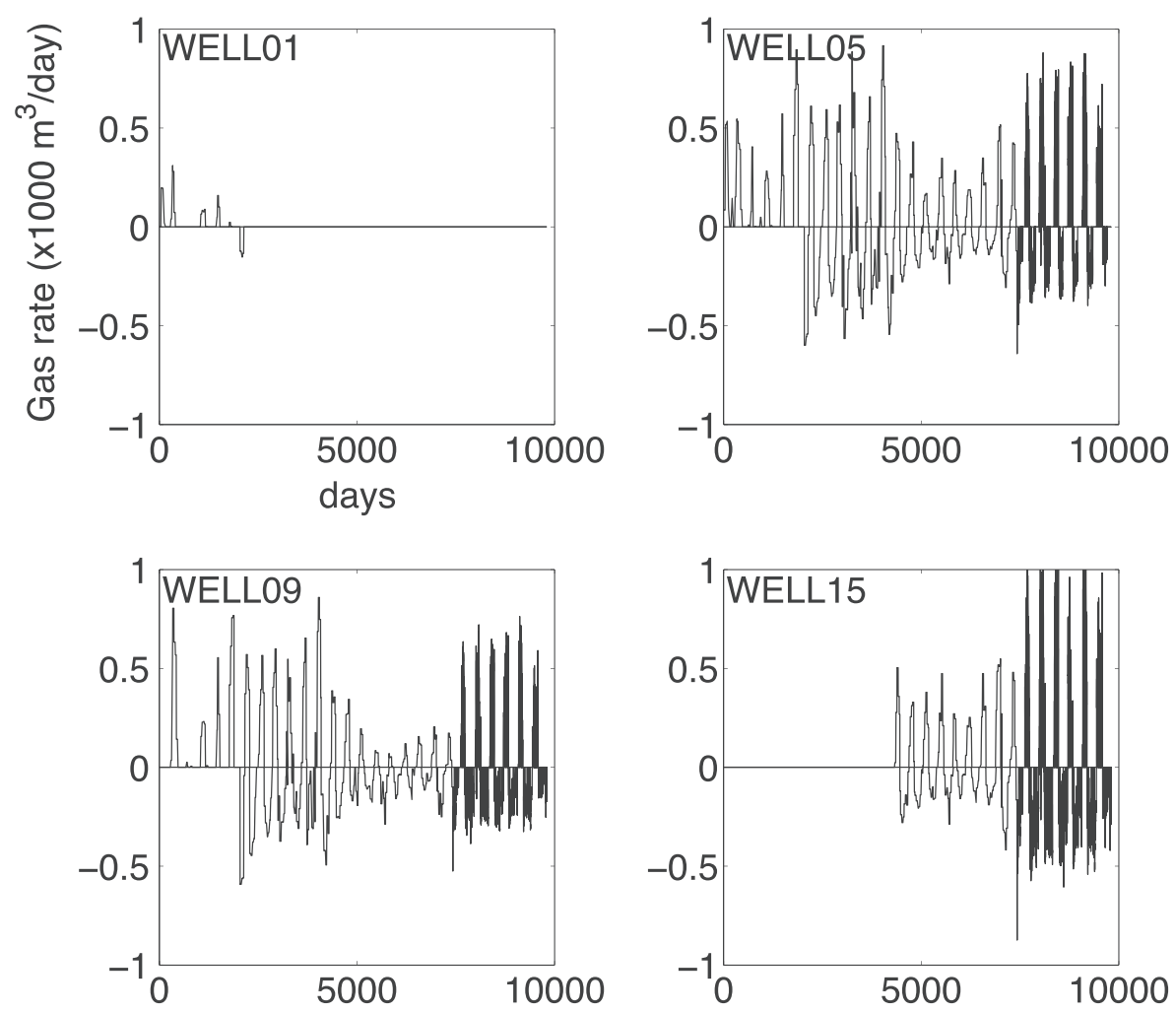

Figure 4. Gas production (positive) and injection (negative) rates of wells in the forward model. Four typical wells are shown. Production volumes are higher than injection volumes.

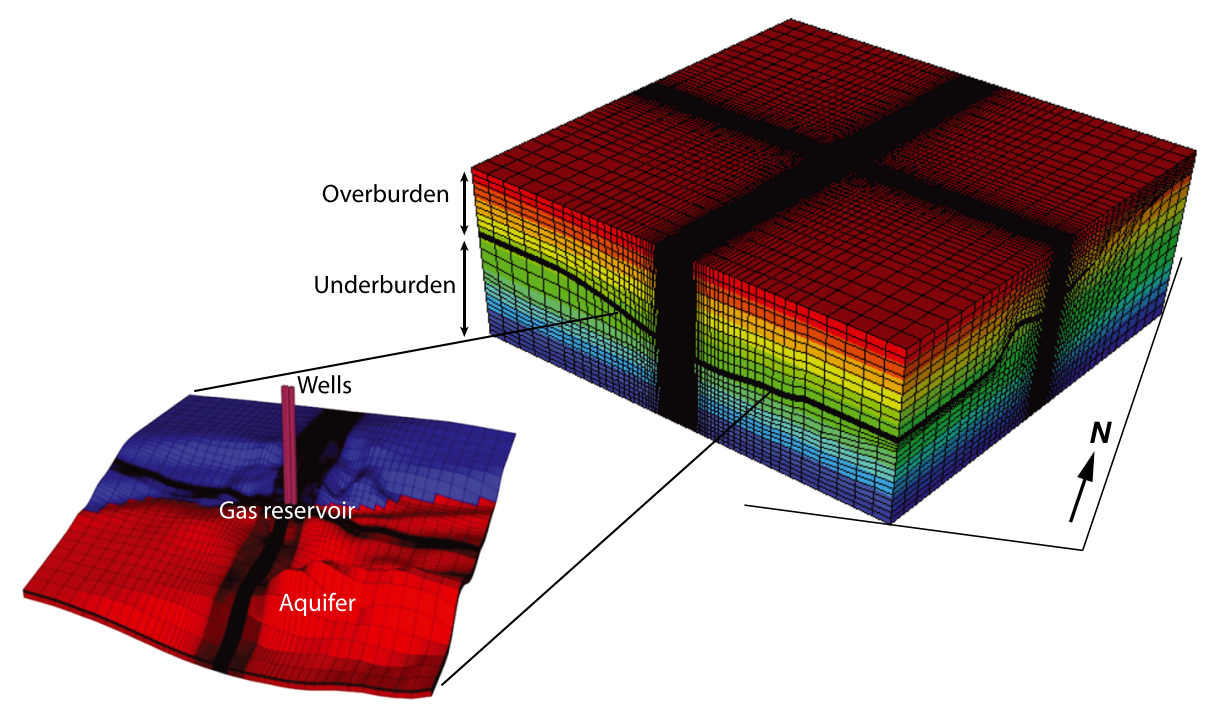

Figure 5. Geomechanical simulation grid (right) and a magnified view of the flow simulation grid (left). The grids are exaggerated by a factor of 7 in the vertical direction. The flow domain appears sandwiched between the overburden and the underburden. The flow domain has two parts, active and inactive, indicated by red and blue colors, respectively. Fluid flow is assumed to occur only in the active part, which is composed of the gas reservoir and the aquifer. Wells are drilled into the gas reservoir.

into 10 and 15 layers, respectively. The finite element grid after discretization is composed of $149 \times 92 \times 35$ elements in the east-west, north-south, and vertical directions, respectively (Figure 5). The geomechanical grid exactly overlaps the flow grid in the flow domain. This allows us to map the pressure data between the two models directly. 
We perform the geomechanical simulation using the software PYLITH, which is a finite element simulator for crustal deformation $[20,46]$. We assume a linear-elastic behavior of the porous medium defined with Young's modulus $E$ and Poisson's ratio $v$. We use a uniform value of Poisson's ratio and a depth-dependent distribution of Young's modulus computed as $E=3(1-2 v)(1+$ $v) / c_{m}(1-v)$, where $c_{m}$ is the uniaxial rock compressibility. There is a relatively large uncertainty in the rock compressibility parameter of the beta field. We use the following relationship to calculate the uniaxial rock compressibility as a function of the vertical stress [47, 48]:

$$
\log c_{m}=a+b \log \sigma_{\text {vert }}^{\prime},
$$

where $c_{m}$ is in $\operatorname{bar}^{-1}, \sigma_{\text {vert }}^{\prime}$ is the effective vertical stress in bar, and $a$ and $b$ are two empirical constants. $\sigma_{\text {vert }}^{\prime}$ is calculated from the total vertical stress $\sigma_{\text {vert }}$ and the pore pressure $p$ using the principle of effective stress, $\sigma_{\text {vert }}^{\prime}=\sigma_{\text {vert }}-\beta p$, with a Biot coefficient $\beta=1$. Here, compressive stresses are positive. We calculate $\sigma_{\text {vert }}$ as a function of depth from a site-specific empirical equation that is based on density logs available as measurements along wells. We calculate $p$ from a hydrostatic pressure profile assumed along the reservoir. Note that we use a pore compressibility in the flow model, $c_{p}=c_{m} / 3 \phi$, which is consistent with the uniaxial compressibility in the geomechanical model [49]. We consider $a$ and $v$ as uncertain parameters in the geomechanical model (Table I) and fix the value of the slope, $b=-1.164$, which is believed to be better constrained than the intercept $a$ [48].

\section{INVERSION METHODOLOGY}

The ensemble-based inversion approach requires a large number of forward model simulations corresponding to different possible realizations of the parameters. In this study, we perform 100 forward model simulations corresponding to 100 realizations. We ran ES for $N=25,50,75,100$ (not shown here), and we find that at $N=100$, the posterior distributions are robust with respect to the ensemble size. We run these simulations over the period of interest of 26 years with an average timestep size of 1 month. The actual timestep size varies with the fluctuating schedule of the imposed well flow rates and convergence requirements within the flow solver. We use the one-way coupled approach [50-55]. In the one-way coupled approach, we first run flow simulations for each realization of porosity, permeability, and pore compressibility using GPRS, followed by poroelastic simulations in PYLITH using pressure fields from the flow simulation and realizations of $a$ and $v$. The coupling between flow and mechanics is provided by the pore pressure, which changes the effective stress in the solution of the momentum balance equation in PYLITH. In this one-way coupled approach, there is no feedback from the mechanics simulation to the flow simulation. This approach is justified for gas reservoirs, where mechanics-to-flow coupling is weak because gas compressibility is usually much larger than rock compressibility [56, 57]. Pressure response of such reservoirs is dominated by gas compressibility, porosity, and permeability in the reservoir and surrounding aquifer and well flow rates.

We list all the inversion parameters of the study in Table I, along with the values used to generate their prior distributions. These values reflect prior information about the parameters obtained from sources such as regional geology, information about the initial state of the system such as initial pressure and saturation, information available in analogous fields, physical constraints, values from literature, and expert opinion. To satisfy the requirements of Gaussian prior statistics for optimality of the Kalman filter-based solution, we transform the uniformly distributed $k v_{r} / k h_{r}$ and $k_{a} / k_{r}$ into normally distributed $\Psi^{-1}\left(k v_{r} / k h_{r}\right)$ and $\Psi^{-1}\left(k_{a} / k_{r}\right)$ parameters, where $\Psi^{-1}$ denotes the inverse of the cumulative normal distribution function with mean and variance of the respective parameter from Table I. We generate an ensemble of 100 realizations by randomly drawing from these prior distributions (Figure 6). We run forward model simulations for each of these realizations to generate the ensemble of prior predictions. It is important for the prediction ensemble to encompass the measurements, and we consider this aspect during the selection of the prior ensemble of parameters.

The inputs to ES are the prior distributions of the inversion parameters, predicted WBHP (bottomhole pressure) for all producing/injecting wells at 200 equally spaced discrete timesteps from all the GPRS runs, and predicted LOS displacements at 2436 ground locations (PSs) at 18 timesteps from 

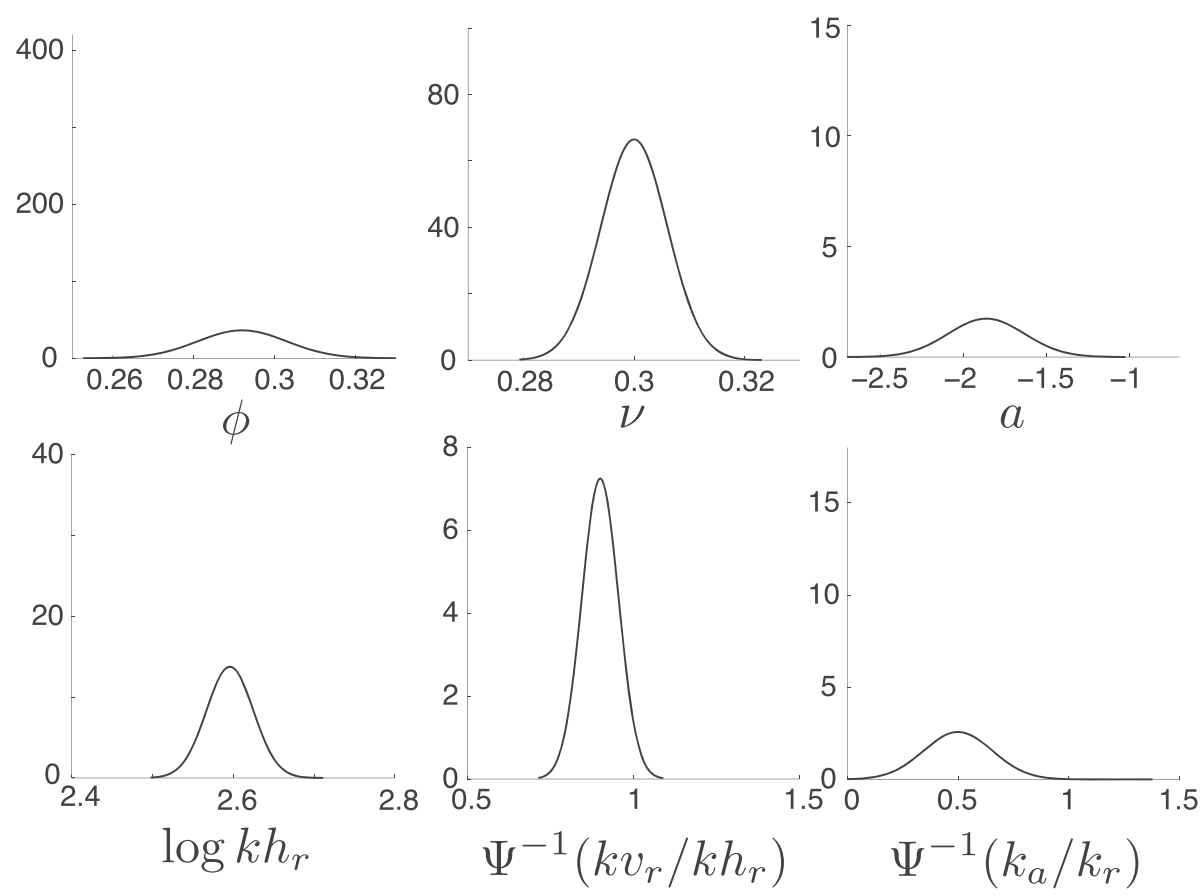

Figure 6. Prior probability density functions of the model parameters generated from values in Table I. $\Psi^{-1}$ denotes the inverse of the cumulative normal distribution function with mean and variance of the respective parameter from Table I.

all the PYLITH runs, measured WBHP and LOS displacements, and the error covariance matrix for all the measurements. In this study, we use WBHP from a history-matched (calibrated to historical measurements) model of the field as our measurement of WBHP in wells. We obtain the predicted LOS displacements from the PYLITH output in three steps: (1) find the PYLITH grid node locations closest to the locations of the PS in the InSAR data; (2) find the PYLITH simulation timesteps closest to the InSAR dates; and (3) convert the PYLITH displacement vectors at these nodes and timesteps into LOS displacements by multiplying with the direction cosines of the ascending track of Envisat: $[-0.3836,-0.1017,0.9179]$ (Figure 1). We used the shortest distance criterion in Step 1 to find the PYLITH node nearest to a PS. The minimum and maximum distance between neighbors thus assigned are 4 and $861 \mathrm{~m}$, respectively.

For inversion using ES, we need to estimate the error covariance of the measurements, $\boldsymbol{C}_{\epsilon \epsilon}$ (Equation (1)). Assuming independence of the individual pressure measurements, we use an identity matrix for the error covariance of the pressure data with a variance of $1 \mathrm{bar}^{2}$. For the error covariance of the processed InSAR data, we use a Gaussian covariance model that is uncorrelated in time, where we assume that the error covariance decays exponentially over the correlation length $L_{c, t}$ of the image at a timestep $t$. That is, the $(i, j)$ element of the displacement part of the error covariance matrix $\boldsymbol{C}_{\epsilon \epsilon}$ in Equation (1) is $\sigma_{t}^{2} \exp \left(-r_{i j}^{2} / L_{c, t}^{2}\right)$, where $r_{i j}$ is the distance between the $i$ th and $j$ th measurement locations and $\sigma_{t}^{2}$ is the variance of processed LOS displacements at time $t$. This results in a block-diagonal error covariance matrix, where blocks are the $2436 \times 2436$ covariance matrices at each of the 18 timesteps.

The output of the ES is the posterior ensemble of the model parameters, which is used to obtain the updated ensemble mean and variance of each model parameter. Using a Gaussian kernel, we construct the continuous versions of posterior distributions from the posterior ensemble. A narrower posterior distribution indicates a reduction in the uncertainty associated with that particular parameter. We run one-way coupled flow and geomechanical simulations with the posterior or updated values of the parameters to assess the agreement between the predicted and measured values of WBHPs and LOS displacements. 


\section{RESU LTS}

The quality of the ensemble Kalman solution depends on the rank and conditioning of the prior ensemble matrix [42]. In our case, when the WBHP and LOS displacements predicted from the prior simulations have trends that are similar to those observed in the respective measurements and the spread in predictions captures the measurements, we expect a high-quality solution from ES that reduces the uncertainty in model parameters. Figure 7 shows that the measured WBHP is captured by the spread in the prior model predictions, and during the pre-UGS period, the predicted pressures overlap the measured pressure. The fluctuations in WBHP during injection or production steps (difference between peak and trough values in Figure 7) are, in general, lower in the prior predictions compared with those in the measurement (Figure 8). Because the flow rates are the same between the predictions and the measurement, this difference in $\triangle$ WBHP between predictions and measurement may suggest that the reservoir permeability, porosity, and/or pore compressibility in the prior ensemble is/are higher than the respective true values.

Figure 9 shows the comparison of the LOS displacement time series from the prior simulations and InSAR measurements at four locations around the gas reservoir, where we have well pressures. In Figure 10, we compare LOS displacements as predicted by one of the prior simulations with the measurements. Prediction and measurement are compared side by side over the area recorded by the satellite. Negative displacement (blue color) means subsidence, which is maximum at the end of winter when most of the gas is produced. Positive displacement (red color) means uplift, which is usually observed at the end of injection during summer months. The discrepancy between predicted and measured displacements is relatively large compared with that in the WBHP data. Most of the discrepancy is observed in the aquifer region outside the gas reservoir. Other than the uncertainty in the model parameters, this discrepancy can be attributed to two sources: (1) noise in the satellite measurements, such as those due to the effect of the atmosphere, and (2) deformation signal in the satellite measurements due to physical features and mechanisms that are not included in the simulation model, such as lateral heterogeneity in petrophysical properties, inelastic deformation, and background deformation from sources outside the study area, like UGS operations in nearby fields or regional subsidence due to groundwater pumping from shallow aquifers. InSAR data processing
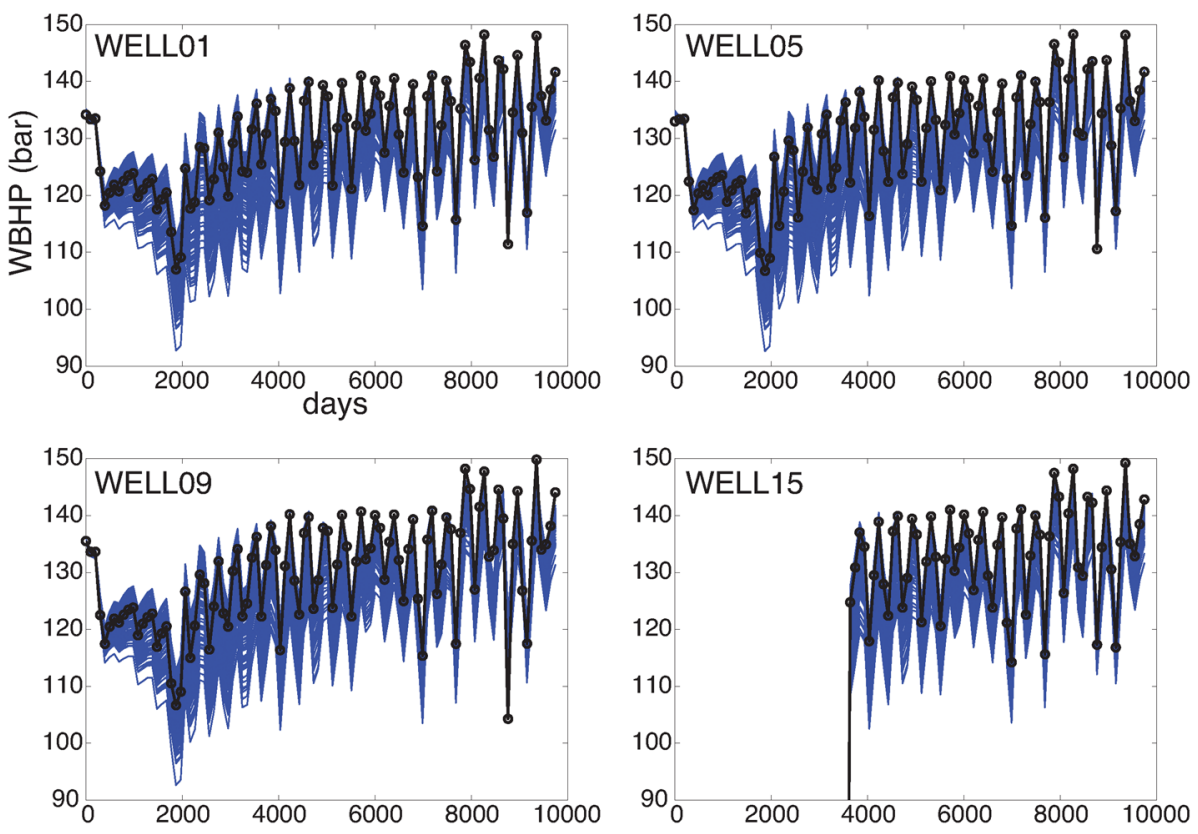

Figure 7. Comparison of the well bottomhole pressure (WBHP) time series from the prior ensemble (blue lines) and from the measurement (black line with circles). Four wells are shown. There are 100 prediction curves corresponding to 100 members of the ensemble. The oscillatory behavior of pressure follows from the seasonal nature of the underground storage program. 

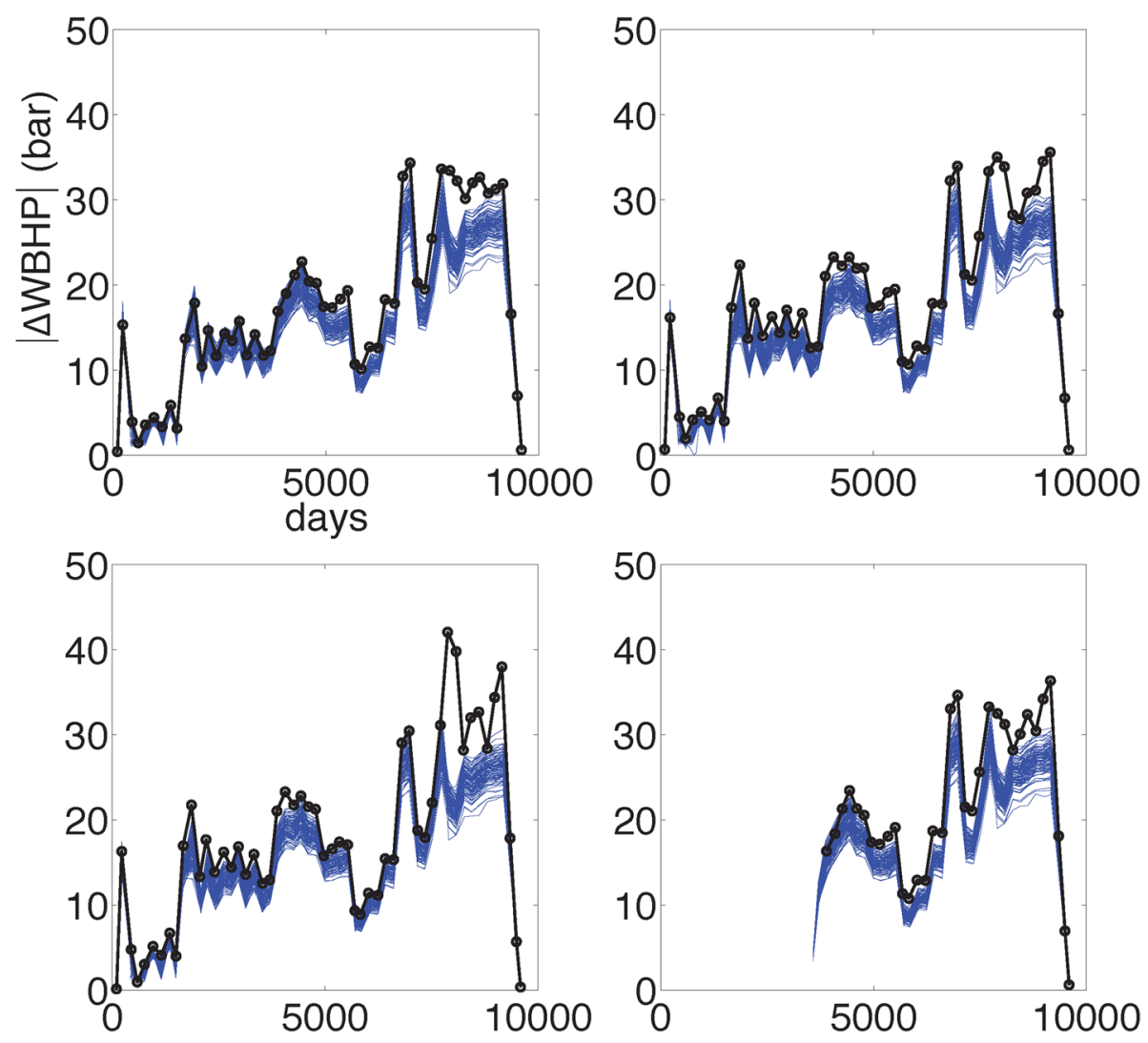

Figure 8. Comparison of the fluctuations in well bottomhole pressure (WBHP) between the prior predictions (blue lines) and the measurement (black line with circles) for four wells. The smaller values of the predicted fluctuations compared with those of the measured fluctuation may suggest a higher value of permeability, porosity, or compressibility in the prior ensemble compared with their true values.

algorithms correct for some of the systematic errors such as the ones due to atmosphere, vegetation, and topography $[25,58,59]$. However, these algorithms have limitations $[26,60]$.

Once we have generated the prior ensemble of predictions, we perform inversion using ES as described in Section 5 discussed earlier. We consider two assimilation scenarios: (1) assimilation of well pressure data and (2) assimilation of both surface displacement and well pressure data. In this way, we can evaluate the benefit of combining surface displacement and well pressure data on parameter estimation and uncertainty reduction.

\subsection{Assimilation of well pressure data}

We use measurement and predictions of the WBHPs to update the model parameters using the ES. The update is conditioned to data in the gas reservoir only because there are no wells in the aquifer. As shown in Figure 7, bottomhole pressures in the prior ensemble are smaller than the measured bottomhole pressure. Given our confidence in the measurement, we expect the updated model parameters to result in a posterior ensemble of bottomhole pressures that is closer to the measurement. Because the well flow rates and gas compressibility are prescribed in the flow simulations, variations in well pressure result from variations in porosity, pore compressibility, reservoir permeability, and aquifer permeability. The first two parameters determine the transient response of the flow domain during fluid storage or drainage of the pores. The last two parameters determine the flux of gas and water through the gas reservoir and the aquifer. The ES can capture these correlations between the parameters and the state variables $\left(\boldsymbol{C}_{\alpha d}\right.$ in Equation (1)) to generate posterior distributions of the parameters (Figure 11) that will result in predicted pressures closer to the measured pressure. 

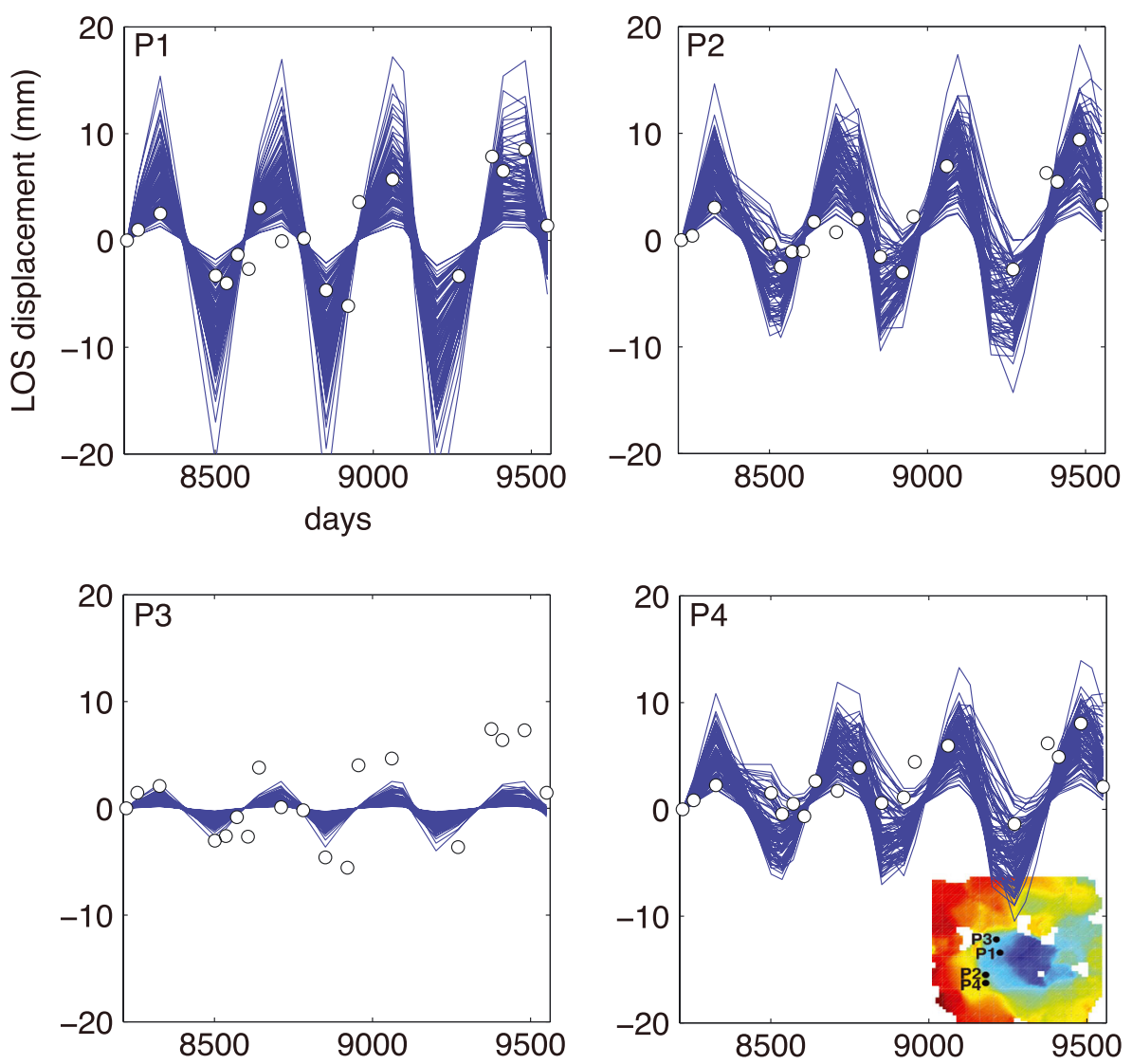

Figure 9. Comparison of the line-of-sight (LOS) displacement time series from the prior ensemble (blue lines) and the interferometric synthetic aperture radar data (white circles) at four locations: P1, P2, P3, and $\mathrm{P} 4$. The locations are marked on the map in the inset. P1 and P2 record higher displacements because they are closer to the wells and the center of the subsidence bowl.

The uncertainty in porosity, compressibility, and reservoir and aquifer permeability is reduced. Poisson's ratio is a parameter in the mechanical model and, therefore, experiences almost no change in its distribution by assimilation of the well pressure data in a one-way coupled forward model. The pore compressibility in the flow model depends on porosity and the rock compressibility parameter $a$, which is a poroelastic coupling parameter influencing both the flow and mechanical response of the system. A higher value of $a$ in the posterior ensemble suggests a higher pore compressibility resulting in a smaller net pressure drop due to net production in the field (Figure 4) and hence higher wellbore pressures. This improves the agreement between the measured pressures and the predicted pressures. Mean porosity decreases in the posterior distribution to honor larger well pressure fluctuations during pre-UGS and UGS periods.

We observe a reduction in the reservoir permeability and an increase in the aquifer-reservoir permeability ratio. The aquifer permeability, which is the product of $k_{r}$ and $k_{a} / k_{r}$, also decreases compared with its prior. This result can be explained as follows. A smaller magnitude of fluctuations in the WBHP prior compared with the measurements (Figure 8) may result from too high reservoir permeability, aquifer permeability, porosity, or pore compressibility in the prior ensemble. The effect of aquifer permeability on the predicted WBHP is weaker than that of the reservoir permeability because the wells are located within the reservoir. Therefore, to honor the measured $|\triangle W B H P|$, the posterior permeabilities are smaller than the prior. However, an interesting result is obtained because of the lack of conditioning in the aquifer. An aquifer permeability higher than the reservoir permeability, $k_{a} / k_{r}>1$, is not consistent with the prior knowledge about the beta field. In the following, we see that conditioning to the surface displacement data, which includes the aquifer region, gives us a result that is consistent with the prior knowledge. 


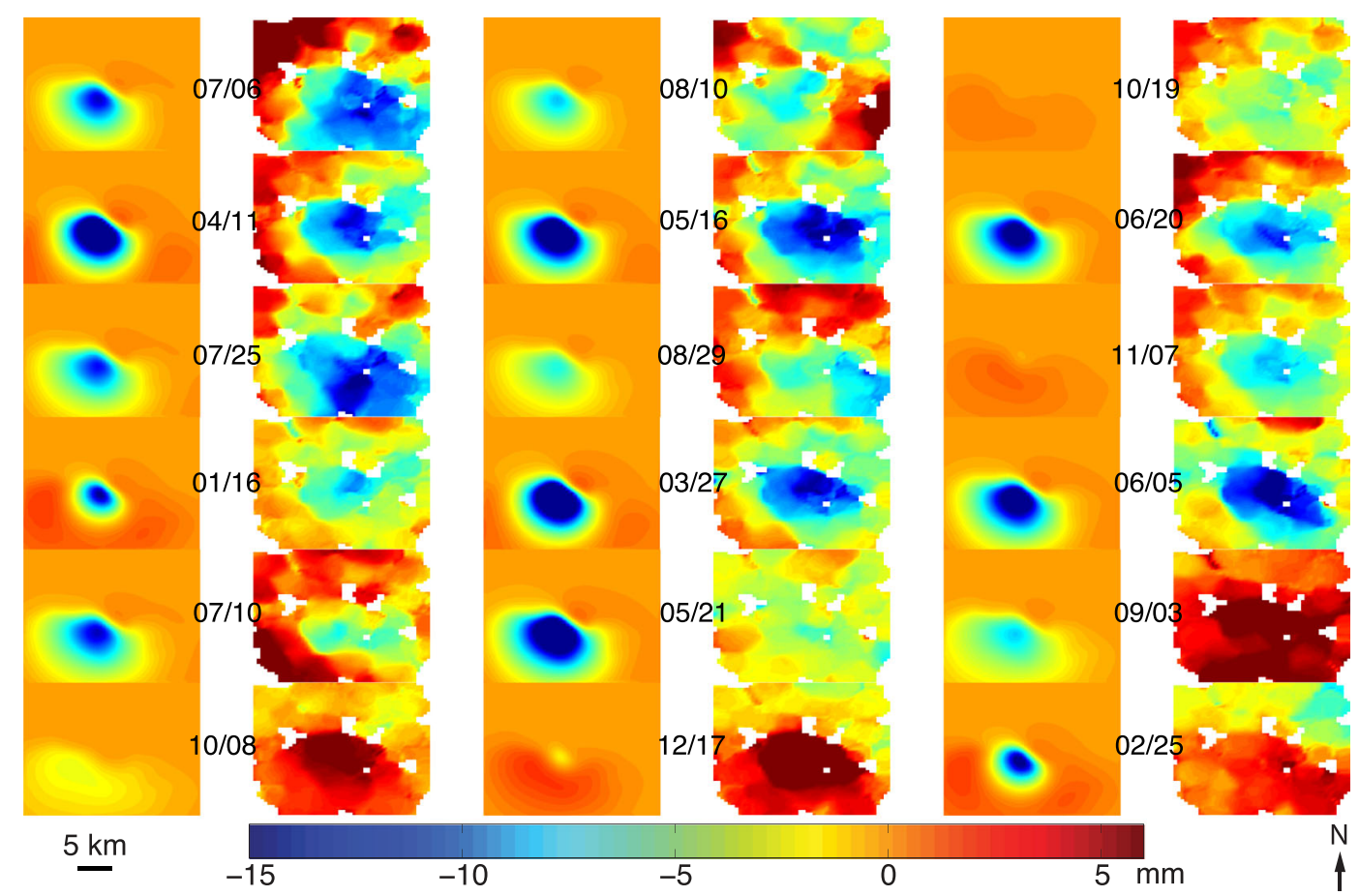

Figure 10. Comparison of the line-of-sight displacement between one member of the prior ensemble (left columns) and the interferometric synthetic aperture radar data (right columns) at 18 successive timesteps. The ensemble member is chosen randomly (it is the 50th realization from the ensemble of 100 realizations). Left and right figures in each pair are separated by the timestep value given in month-day format. The timesteps increase from left to right and then top to bottom. The gaps in the interferometric synthetic aperture radar map correspond to missing data at those locations. Presence of a blue-colored subsidence bowl above the gas reservoir during gas production periods is evident.

\subsection{Assimilation of interferometric synthetic aperture radar and well data}

We use both the InSAR displacement data and the well pressure data to update the model parameters using ES. As shown in Figure 10, measured displacements are, in general, higher than the predicted displacements, especially outside the gas reservoir where the model lacks information about the petrophysical properties (model parameters) and any extraneous sources of deformation, if present. The surface displacements from the mechanical model are determined by pressure fluctuations in the reservoir and elastic properties of the medium, that is, the rock compressibility and Poisson's ratio. An improved agreement between the measured and predicted displacements requires larger pressure fluctuations, whereas an improved agreement between the measured and predicted well pressures requires larger pressure fluctuations and higher absolute pressures in the system. The posterior distributions of the model parameters (Figure 11) reflect this balance between the two physical mechanisms of the coupled system, mechanical deformation and fluid flow, because of conditioning to the two types of measurements, displacement and pressure.

We observe a reduction in the uncertainty of the aquifer-reservoir permeability ratio $k_{a} / k_{r}$, compressibility parameter $a$, and porosity $\phi$. More importantly, assimilation of both the InSAR and the well data results into posterior distributions of these parameters, which are more reliable than those obtained by assimilation of the pressure data alone. For example, assimilation of the InSAR and well data results in $k_{a} / k_{r}<1$, which agrees with the expected behavior in the field, and mean of $a$ closer to its prior mean compared with the scenario when only the pressure data are assimilated. The posterior distribution of $k_{a} / k_{r}$ is narrower and centered near the upper bound in its prior. A higher aquifer permeability is necessary to transmit the reservoir pressure fluctuations into the aquifer and thereby honor the large displacements observed in the InSAR data outside the gas reservoir (Figure 10). The reservoir permeabilities $\left(k v_{r}, k h_{r}\right)$ are similar to their prior values because of 

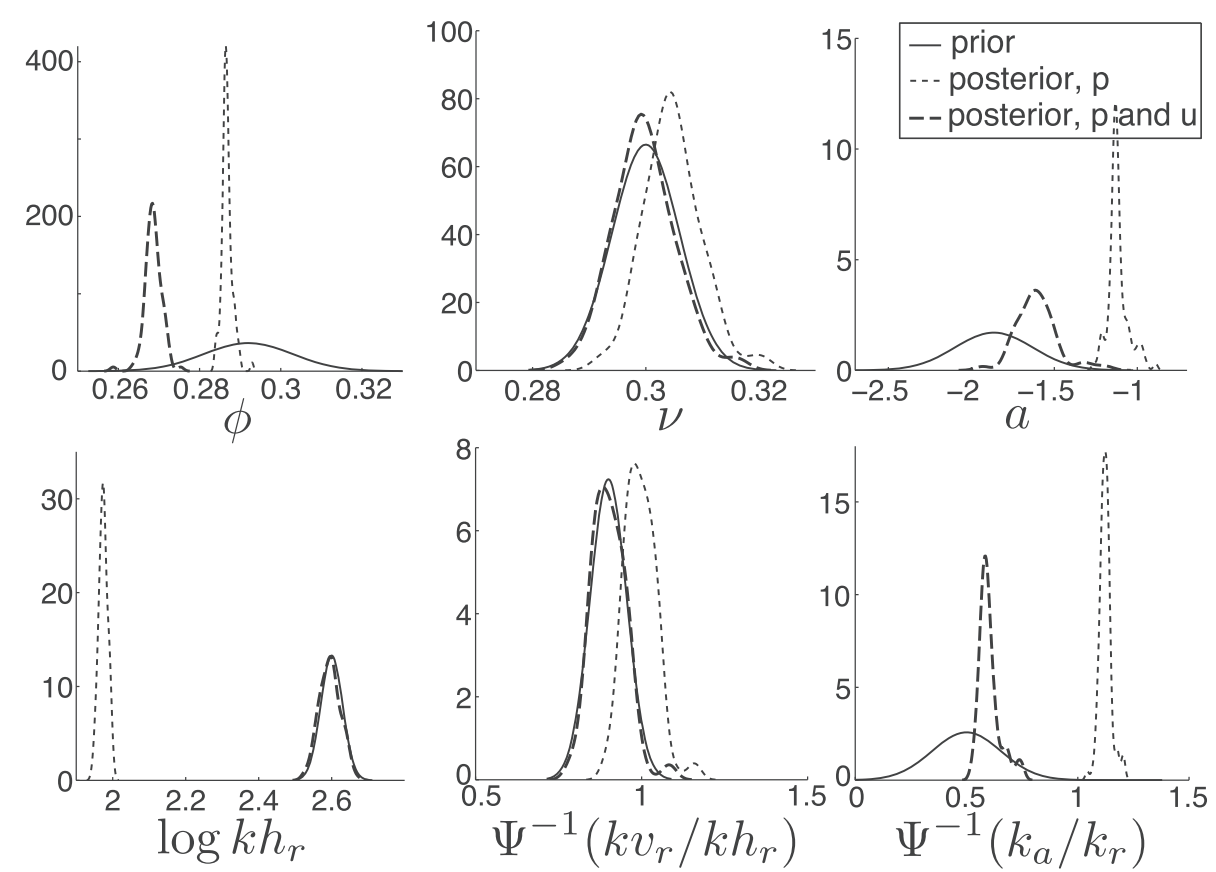

Figure 11. Comparison of the prior (solid lines) and posterior distributions of the model parameters with inversion performed using only the well pressure data (thin dash lines) and using both the interferometric synthetic aperture radar data and the well pressure data (thick dash lines). Posterior distributions are approximated from the posterior ensemble of the parameters using a Gaussian kernel.
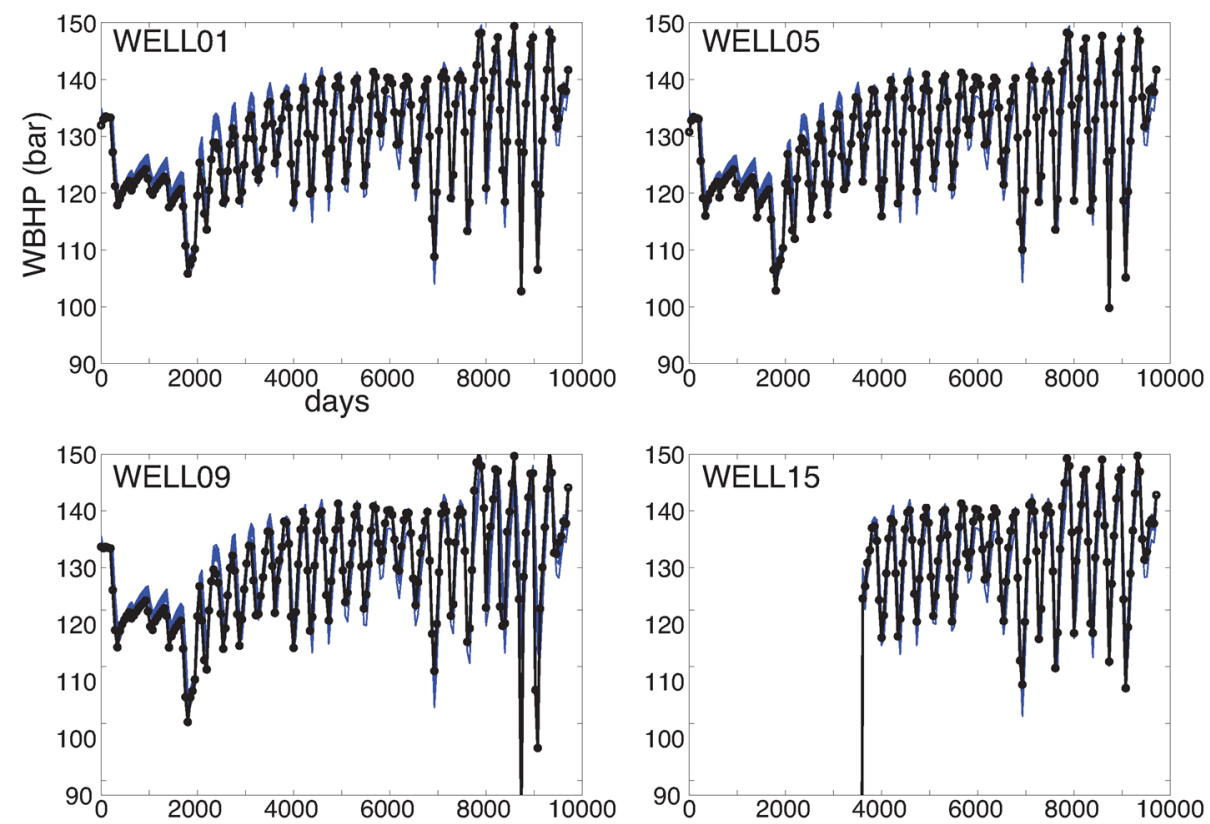

Figure 12. Excellent agreement between the well bottomhole pressure (WBHP) time series from the posterior ensemble (blue lines) and from the measurement (black line with circles). Four wells are shown. There are 100 prediction curves, overlapped on each other, corresponding to 100 members of the ensemble.

the balancing effects of the displacement and pressure data. Also, the prior uncertainty in the reservoir permeability is already small. Mean porosity decreases to honor larger pressure fluctuations in the measurement. We conclude that joint inversion using both surface deformation and well data 
reduces the uncertainty in two relatively unknown parameters in the beta field: the strength of the aquifer and the rock compressibility.

We assess the performance of the ES by comparing the posterior predictions of WBHP and LOS displacements with their respective measurements. We generate the predictions by performing oneway coupled flow and geomechanical simulations with 100 members of the posterior ensemble. Figure 12 shows that the posterior prediction of the WBHP has, indeed, improved drastically, as all the prediction curves cluster together around the measurement curve. This result illustrates the robustness of the inversion methodology, given that the prior ensemble of WBHP had a tendency to underpredict the measured WBHP (Figure 7). Similarly, we see a reduction in the spread of the predicted displacement curves in Figure 13 compared with the prior ensemble in Figure 9. Because the surface displacement is proportional to the product of the pressure perturbation, rock compressibility, and Poisson's ratio, the uncertainty in predicted displacement combines the uncertainty in the pressure and the elastic properties. Therefore, we observe a larger spread in the displacement ensemble compared with that in the pressure ensemble. Note that, in the one-way coupled approach followed here, pressure is not a function of displacement. Comparing Figure 14 with Figure 10, we also observe that the posterior displacements are larger in magnitude than the prior displacements. This results from larger InSAR displacements recorded in the region outside the gas reservoir, for example in the aquifer.
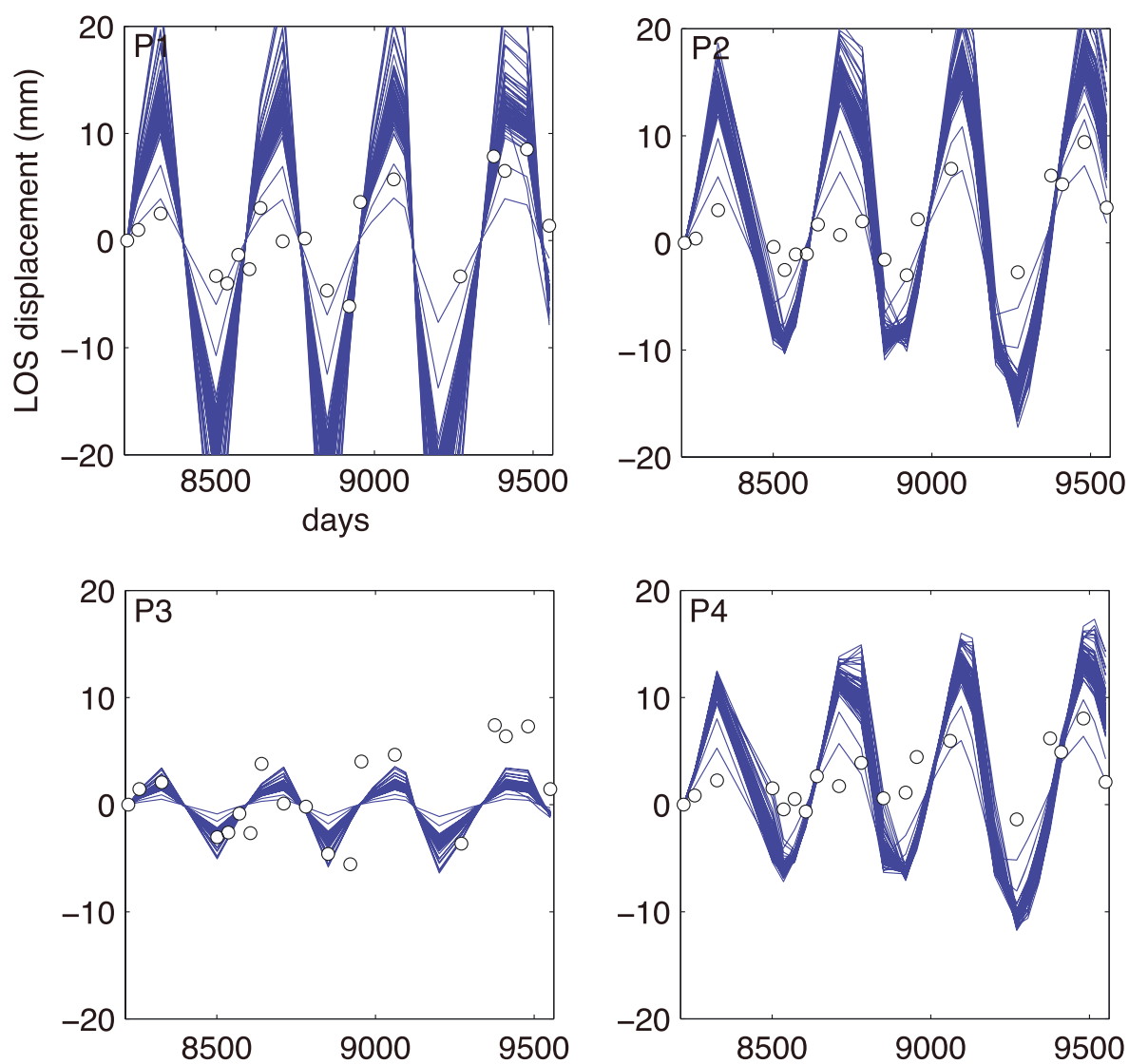

Figure 13. Comparison of the line-of-sight (LOS) displacement time series from the posterior ensemble (blue lines) and the interferometric synthetic aperture radar data (white circles) at four locations. The variability in the posterior ensemble (spread of the blue curves) has decreased compared with that in the prior ensemble (Figure 9). To honor the large displacements in the interferometric synthetic aperture radar data outside the gas reservoir (Figure 10), ES updates the model parameters such that the posterior displacements are larger in magnitude than the prior displacements. 


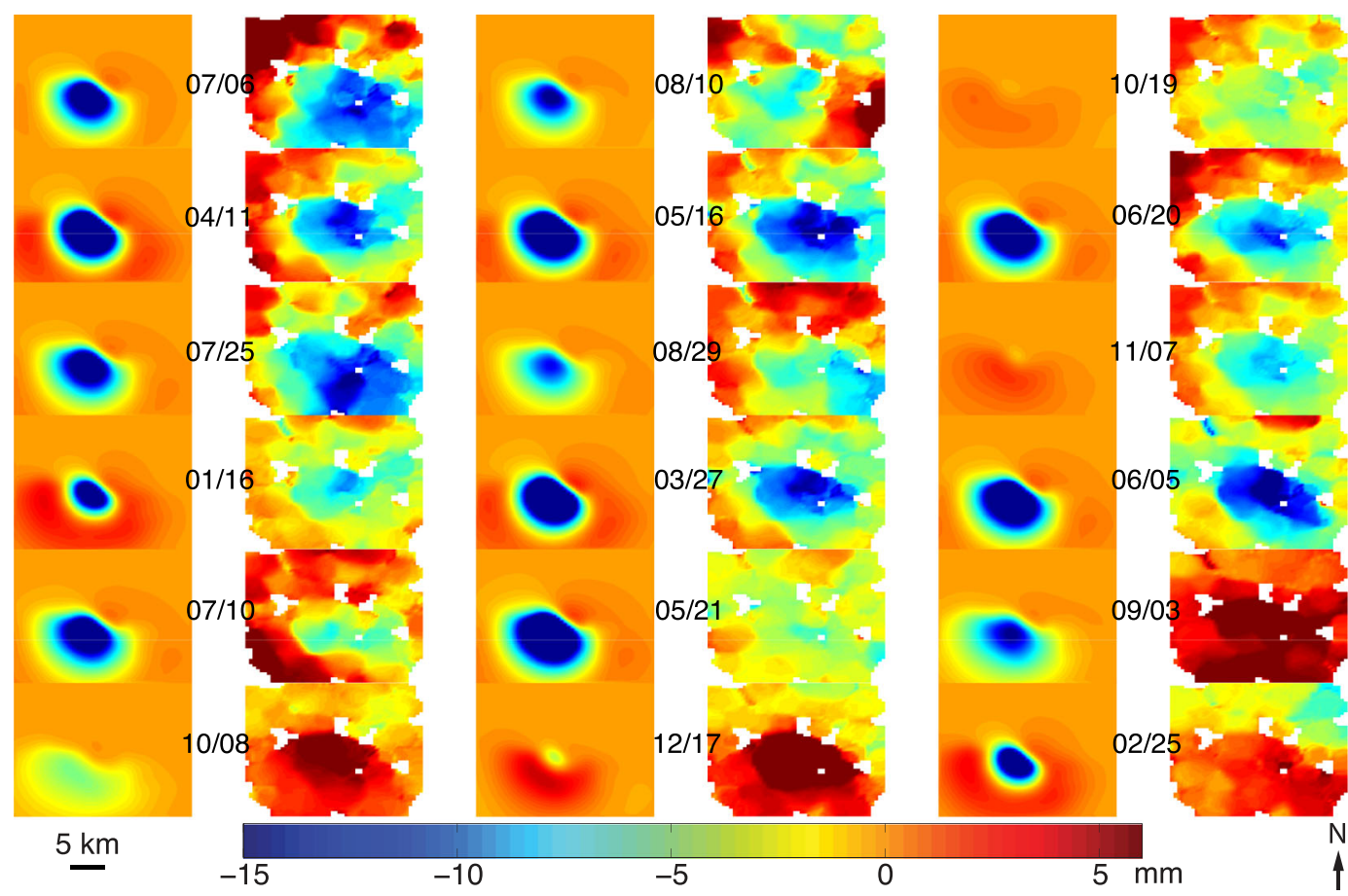

Figure 14. Comparison of the line-of-sight displacement between one member of the posterior ensemble (left columns) and the interferometric synthetic aperture radar data (right columns) at 18 successive timesteps. The ensemble member is chosen randomly (it is the 50th realization from the ensemble of 100 realizations). Left and right figures in each pair are separated by the timestep value given in month-day format.

\section{DISCUSSION AND CONCLUSIONS}

We perform a probabilistic estimation of rock properties by joint inversion of ground deformation data from geodetic measurements and fluid flow data from wells. Using an efficient implementation of the ES as the estimator and our coupled multiphase flow and geomechanics simulator as the forward model, we conclude that assimilation of flow and deformation data leads to reduction of uncertainty in the prior distributions of rock properties such as porosity, permeability, and compressibility. We show that our method can reduce uncertainty over both the reservoir region, where prior information is available from wells, and the aquifer region, where there are no wells. This is an important result in the case of the beta field.

It may be possible to further improve the impact of ground deformation data on uncertainty reduction by improving the InSAR data processing. In this study, we used data from the ascending tracks of Envisat. We can assess the impact of merging both the ascending and descending track datasets on uncertainty reduction [12]. Further, the error covariance analysis and smoothing of the InSAR data can be improved to increase the weight (inverse of the error covariance matrix) of the ground deformation data in the Kalman gain matrix of ES.

We are also interested in improving the coverage of the displacement data by enriching the spatially continuous (but noisy) InSAR dataset with temporally continuous GPS measurements. This is particularly useful for the area above the aquifer because InSAR data currently show higher noise in this area. GPS data complement the InSAR data because these can act as a source of independent measurement that is time continuous, absolute, and three-dimensional instead of being restricted to the LOS direction [61, 62]. GPS data can also be used to estimate the atmospheric contribution to the InSAR signal, which is usually a large source of uncertainty during InSAR processing [24, 63].

We used a history-matched flow simulation as our source of pressure measurements. In a subsequent study, we will investigate the issue of any bias introduced by the history-matching process, and we will account for such bias by modifying the measurement error covariance matrix of the pressure data. 


\section{ACKNOWLEDGEMENTS}

Funding for this work was provided by Eni S.p.A. through the Multiscale Reservoir Science Project.

\section{REFERENCES}

1. Oliver DS, Chen Y. Recent progress on reservoir history matching: a review. Computers \& Geosciences 2011; 15:185-221.

2. Gavalas GR, Shah PC, Seinfeld JH. Reservoir history matching by Bayesian estimation. Society of Petroleum Engineers Journal 1976; 16:337-350.

3. Yeh WWG. Review of parameter identification procedure in groundwater hydrology: the inverse problem. Water Resources Research 1986; 22:85-92.

4. Lumley DE. Time-lapse seismic reservoir monitoring. Geophysics 2001; 66:50-53.

5. Hyndman DW, Gorelick SM. Estimating lithologic transport properties in three dimensions using seismic and tracer data: the Kesterson aquifer. Water Resources Research 1996; 32:2659-2670.

6. Huang X, Meister L, Workman R. Reservoir characterization by integration of time-lapse seismic and production data. SPE Annual Technical Conference and Exhibition, SPE 38695, San Antonio, TX, 1997. DOI: $10.2118 / 49146-M S$.

7. Vasco DW, Datta-Gupta A, He Z. Reconciling time-lapse seismic and production data using streamline models: the Bay Marchand field, Gulf of Mexico. SPE Annual Technical Conference and Exhibition, SPE 84568, Denver, CO, 2003. DOI: $10.2118 / 84568-M S$.

8. Wen X H, Lee S, Yu T. Simultaneous integration of pressure, water cut, and 4-D seismic data in geostatistical reservoir modeling. Mathematical Geosciences 2006; 38:301-325.

9. Galloway DL, Hudnut K, Ingebritsen S, Phillips S, Peltzer G, Rogez F, Rosen PA. Detection of aquifer system compaction and land subsidence using interferometric synthetic aperture radar, Antelope Valley, Mojave Desert, California. Water Resources Research 1998; 34:2573-2585.

10. Galloway DL, Hoffmann J. The application of satellite differential SAR interferometry-derived ground displacements in hydrogeology. Hydrogeology Journal 2007; 15:133-154.

11. Vasco DW, Rucci A, Ferretti A, Novali F, Bissell RC, Ringrose PS, Mathieson AS, Wright IW. Satellite-based measurements of surface deformation reveal fluid flow associated with the geological storage of carbon dioxide. Geophysical Research Letters 2010; 37:3071-3089.

12. Teatini P, Castelletto N, Ferronato M, Gambolati G, Janna C, Cairo E, Marzorati D, Colombo D, Ferretti A, Bagliani A, Bottazzi F. Geomechanical response to seasonal gas storage in depleted reservoirs: a case study in the Po River basin, Italy. Journal of Geophysical Research 2011; 116:F02002.

13. Gonzalez PJ, Tiampo KF, Palano M, Cannavo F, Fernandez J. The 2011 Lorca earthquake slip distribution controlled by groundwater crustal unloading. Nature Geoscience 2012; 5:821-825.

14. Vasco D, Karasaki K, Kishida K. A coupled inversion of pressure and surface displacement. Water Resources Research 2001; 37:3071-3089.

15. Vasco D. Estimation of flow properties using surface deformation and head data: a trajectory-based approach. Water Resources Research 2004; 40:W10104.

16. Iglesias MA, McLaughlin D. Data inversion in coupled subsurface flow and geomechanics models. Inverse Problems 2012; 28:115009.

17. Hesse MA, Stadler G. Joint inversion in coupled quasi-static poroelasticity. Journal of Geophysical Research 2014; 119:1425-1445.

18. Chang H, Chen Y, Zhang D. Data assimilation of coupled fluid flow and geomechanics using the ensemble Kalman filter. Society of Petroleum Engineers Journal 2010; 15:1-13.

19. Wilschut F, Peters E, Visser K, Fokker PA, van Hooff PME. Joint history matching of well data and surface subsidence observations using the ensemble Kalman filter: a field study. SPE Reservoir Simulation Symposium, SPE 141690, TX, USA, 2011. DOI: 10.2118/141690-MS.

20. Jha B, Juanes R. Coupled multiphase flow and poromechanics: a computational model of pore-pressure effects on fault slip and earthquake triggering. Water Resources Research 2014; 50(5):3776-3808.

21. Curlander J, McDonough RN. Synthetic Aperture Radar: Systems and Signal Processing. Wiley: New York, 1991.

22. Hooper A, Zebker H, Segall P, Kampes B. A new method for measuring deformation on volcanoes and other natural terrains using InSAR persistent scatterers. Geophysical Research Letters 2004; 31:L23611.

23. Tele-Rilevamento Europa srl. Measuring surface motion. (Available from: http://treuropa.com/technique/) [Accessed on 23 January 2015].

24. Herring TA, Corey BE, Counselman CC, Shapiro II, Ronnang BO, Rydbeck OEH, Clark TA, Coates RJ, Ma C, Ryan JW, Vandenberg NR, Hinteregger HF, Knight CA, Rogers AEE, Whitney AR, Robertson DS, Schupler BR. Geodesy by radio interferometry: intercontinental distance determinations with subdecimeter precision. Journal of Geophysical Research 1981; 86:1647-1651.

25. Davis JL, Herring TA, Shapiro II, Rogers AEE, Elgered G. Geodesy by radio interferometry: effects of atmospheric modeling errors on estimates of baseline length. Radio Science 1985; 20:1593-1607.

26. Hanssen RF. Radar Interferometry: Data Interpretation and Error Analysis. Kluwer Academic: Dordrecht, The Netherlands, 2001. 
27. Doin MP, Lasserre C, Peltzer G, Cavalie O, Doubre C. Corrections of stratified tropospheric delays in SAR interferometry: validation with global atmospheric models. Journal of Applied Geophysics 2009; 69:35-50.

28. Evensen G. Data Assimilation: The Ensemble Kalman Filter. Springer: Berlin, 2007.

29. Aanonsen SI, Nævdal G, Oliver DS, Reynolds AC, Valles B. The ensemble Kalman filter in reservoir engineering: a review. Society of Petroleum Engineers Journal 2009; 14:393-412.

30. Evensen G. Advanced data assimilation for strongly nonlinear dynamics. Monthly Weather Review 1997; 125: 1342-1354.

31. Evensen G. An ensemble Kalman smoother for nonlinear dynamics. Monthly Weather Review 2000; 128:1852-1867.

32. Kalman RE. A new approach to linear filtering and prediction problems. Journal of Basic Engineering 1960; 82: 35-45.

33. Daley R. Atmospheric Data Analysis. Cambridge University Press: New York, 1991.

34. Lewis JM, Lakshmivarahan S, Dhall S. Dynamic Data Assimilation: A Least Squares Approach (Encyclopedia of Mathematics and Its Applications). Cambridge University Press: Cambridge, 2006.

35. Burgers G, van Leeuwen PJ, Evensen G. Analysis scheme in the ensemble Kalman filter. American Meteorological Society 1998; 126:1719-1724.

36. Reichle RH, McLaughlin DB, Entekhabi D. Hydrologic data assimilation with the ensemble Kalman filter. American Meteorological Society 2002; 130:103-114.

37. Nævdal G, Johnsen LM, Aanonsen SI, Vefring EH. Reservoir monitoring and continuous model updating using the ensemble Kalman filter. Society of Petroleum Engineers Journal 2005; 10(1):66-74.

38. Liu Y, Gupta HV. Uncertainty in hydrologic modeling: toward an integrated data assimilation framework. Water Resources Research 2007; 43:W07401.

39. van Leeuwen P, Evensen G. Data assimilation and inverse methods in terms of a probabilistic formulation. Monthly Weather Review 1996; 124:2898-2913.

40. Skjervheim JA, Evensen G, Hove J, Vabo JG. An ensemble smoother for assisted history matching. Reservoir Simulation Symposium, SPE 141929, TX, USA, 2011. DOI: 10.2118/141929-MS.

41. Evensen G. The ensemble Kalman filter: theoretical formulation and practical implementation. Ocean Dynamics 2003; 53:343-367.

42. Evensen G. Sampling strategies and square root analysis schemes for the EnKF. Ocean Dynamics 2004; 54:539-560.

43. Anderson JL. An ensemble adjustment Kalman filter for data assimilation. Monthly Weather Review 2004; 129: 2884-2903.

44. Halko N, Martinsson PG, Tropp JA. Finding structure with randomness: probabilistic algorithms for constructing approximate matrix decompositions. SIAM Review 2011; 53:217-288.

45. Pan H, Cao H. User Manual for General Purpose Research Simulator. Stanford University Petroleum Engineering Institute: Stanford, CA, 2010.

46. Aagaard BT, Knepley MG, Williams CA. A domain decomposition approach to implementing fault slip in finiteelement models of quasi-static and dynamic crustal deformation. Journal of Geophysical Research 2013; 118: 3059-3079.

47. Bau D, Ferronato M, Gambolati G, Teatini P. Basin-scale compressibility of the northern adriatic by the radioactive marker technique. Geotechnique 2002; 52:605-616.

48. Hueckel T, Cassiani G, Prevost JH, Walters DA. Field derived compressibility of deep sediments of the Northern Adriatic. In Land Subsidence, Special Volume Multi-disciplinary Assessment of Subsidence in the Ravenna Area. Millpress: Rotterdam, 2005; 35-51.

49. Geertsma J. The effect of fluid pressure decline on volumetric change of porous rocks. Transactions of AIME 1957; 210:331-340.

50. Settari A, Mourits F. A coupled reservoir and geomechanical simulation system. Journal of Petroleum Science and Engineering 1998; 3:219-226.

51. Settari A, Walters DA. Advances in coupled geomechanical and reservoir modeling with applications to reservoir compaction. Journal of Petroleum Science and Engineering 2001; 6:334-342.

52. Minkoff SE, Stone CM, Bryant S, Peszynska M, Wheeler MF. Coupled fluid flow and geomechanical deformation modeling. Journal of Petroleum Science and Engineering 2003; 38:37-56.

53. Tran D, Settari A, Nghiem L. New iterative coupling between a reservoir simulator and a geomechanics module. Society of Petroleum Engineers Journal 2004; 9(3):362-369.

54. Jha B, Juanes R. A locally conservative finite element framework for the simulation of coupled flow and reservoir geomechanics. Acta Geotechnica 2007; 2:139-153.

55. Samier P, Onaisi A, Gennaro S. A practical iterative scheme for coupling geomechanics with reservoir simulation. SPE Reservoir Evaluation \& Engineering 2008; 11(5):892-901.

56. Wang HF. Theory of Linear Poroelasticity with Applications to Geomechanics and Hydrogeology. Princeton University Press: Princeton, New Jersey, 2000.

57. Gambolati G, Teatini P, Bau D, Ferronato M. The importance of poro-elastic coupling in dynamically active aquifers of the Po river basin, Italy. Water Resources Research 2000; 36:2443-2459.

58. Gabriel AK, Goldstein RM, Zebker HA. Mapping small elevation changes over large areas: differential radar interferometry. Journal of Geophysical Research 1989; 94:9183-9191.

59. Zebker HA, Rosen PA, Goldstein RM, Gabriel A, Werner CL. On the derivation of coseismic displacement fields using differential radar interferometry: the Landers earthquake. Journal of Geophysical Research 1994; 99: 19617-19634. 


\section{B. JHA ET AL.}

60. Zebker HA, Rosen PA, Hensley S. Atmospheric effects in interferometric synthetic aperture radar surface deformation and topographic maps. Journal of Geophysical Research 1997; 102:7547-7563.

61. Dieterich JH, Decker RW. Finite element modeling of surface deformation associated with volcanism. Journal of Geophysical Research 1975; 80:4094-4102.

62. Vasco DW, Jr CW, Karasaki K, Marques O. Geodetic imaging: reservoir monitoring using satellite interferometry. Geophysical Journal International 2002; 149:555-571.

63. Williams S, Bock Y, Fang P. Integrated satellite interferometry: tropospheric noise, GPS estimates and implications for interferometric synthetic aperture radar products. Journal of Geophysical Research 1998; 1032:27051-27068. 